CORRECTION

Check for updates

\title{
Correction to: LNC942 promoting METTL14-mediated m 6 A methylation in breast cancer cell proliferation and progression
}

Tong Sun, Zhikun Wu, Xiufang Wang, Yilin Wang, Xiaoyun Hu, Wenyan Qin, Senxu Lu, Dongping Xu, Yutong Wu, Qiuchen Chen, Xiangyu Ding, Hao Guo, Yalun Li, Yuanhe Wang, Boshi Fu, Weifan Yao, Minjie Wei (D) and Huizhe Wu (iD)

(c) The Author(s), under exclusive licence to Springer Nature Limited 2022

Oncogene (2022) 41:1677; https://doi.org/10.1038/s41388-022-02194-0

Correction to: Oncogene https://doi.org/10.1038/s41388-020-1338-9, published online 23 June 2020

Following the publication of this article, the authors noted misplaced images in Figures $3 \mathrm{~d}$ and $4 \mathrm{e}$.
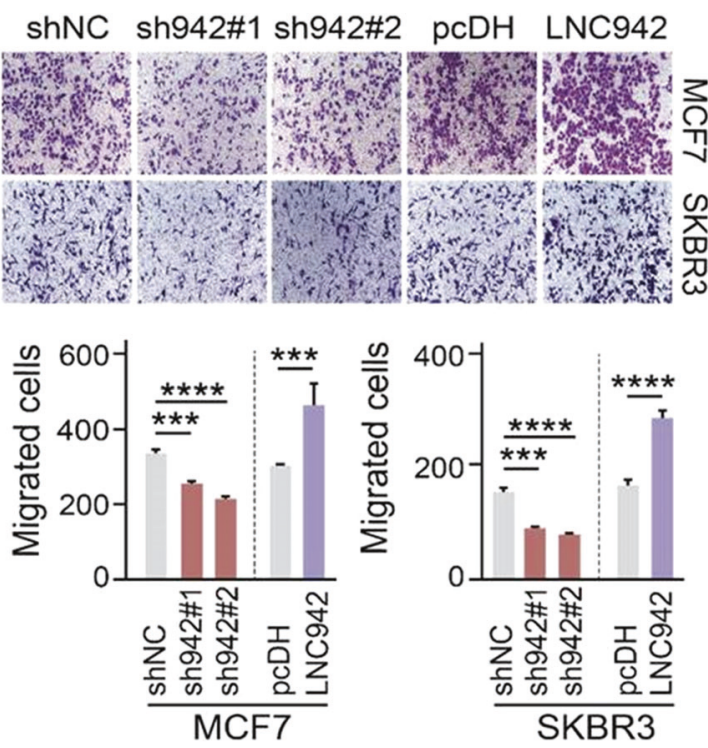

These figures have been updated with the correct images added,

The authors confirm this change has no effect on the conclusions of the study.

Images below:
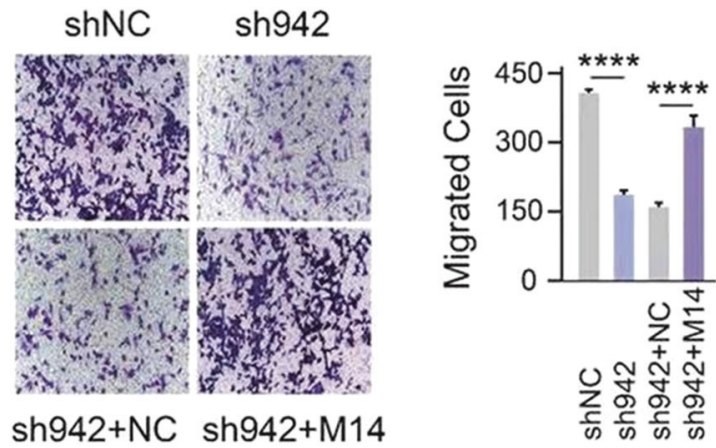

Fig. $4 \mathrm{e}$

The original article has been corrected.

Fig. 3d 